\title{
A filtering approach to the two-dimensional volume conductor forward and inverse problems
}

\author{
Thomas G Xydis $\dagger$, Andrew E Yagle† and Alan H Kadish $\ddagger$ \\ t Department of Electrical Engineering and Computer Science, University of Michi- \\ gan, Ann Arbor, MI 48109-2122, USA \\ $\ddagger$ Department of Internal Medicine, The University of Michigan, Ann Arbor, MI \\ 48109-2122, USA
}

Received 7 December 1990

\begin{abstract}
The two-dimensional layered volume conductor forward and inverse problems are solved by modelling the medium as an equivalent two-dimensional filter relating the source and measured potentials. This extends previous results to the multi-dimensional multi-layered case. In principle, the inverse problem can be solved as easily as the forward problem, using $2 \mathrm{D}$ FFT algorithms. In fact, it is ill-conditioned and must be regularized. We show that Tikhonov regularization, constrained leastsquares regularization, and stochastic regularization using a Wiener filter all lead to the same spatial low-pass regularizing filter. New contributions of this paper include: (1) extension of the medium filter concept from one to two dimensions; (2) a simple layer-recursive formula for computing the medium filter for a layered medium; and (3) application of various regularization techniques, in the form of regularizing filters, to this problem. Numerical simulations are presented which show that conductivity discontinuities have a significant effect on the potential in the volume conductor.
\end{abstract}

\section{Introduction}

\subsection{Problem statement}

The two-dimensional volume conductor forward and inverse problems may be formulated as follows. Given an electrically active sheet or plane at the bottom of a inhomogeneous layered conducting medium, the forward problem is to determine the resulting potential distribution in the surrounding medium. The inverse problem is to determine the potential distribution on the sheet from measurements made in the surrounding tissue. In biomedical applications, the electrically active sheet represents a layer of electrically active cells, and the conducting medium represents interstitial fluid or other electrically inert tissue.

Recent research in cardiology [1-4] has generated interest in volume conductor solutions in rectangular coordinates. This cardiac research is concerned with the propagation anomalies which result from damaged sections of otherwise healthy cardiac tissue. Typically, potential measurements made at the surface of the heart are used to infer the surface potential of the buried active tissue. A detailed mapping of the potentials at the surface of cardiac cells can be used to determine the point of activation, useful in the study of arrhythmias $[1,2,5,6]$. 
The computation of such potentials non-invasively is desirable, since features obscured by the volume conductor (e.g. cell-to-cell propagation irregularities [1]) can then be distinguished more clearly. Furthermore, even in situations where the electrically active cell can be monitored directly, there is a thin layer of conducting fluid between the cell surface and the test electrodes. It will be shown that even thin conducting layers can significantly distort the measured potential.

Other techniques, such as the boundary element technique, have the advantage of allowing the use of more arbitrary geometries. The motivation of our work is to obtain computationally efficient procedures for relating potentials within the ventricular muscle, a few mm from the pericardium, to measured potentials on or very near the surface of the pericardium. Although the surface of the heart is not a plane, it is reasonable to consider it to be planar over such a localized region, due to the rapid attenuation caused by volume conductors even at small distances. The planar heart approximation has been used in the past for the volume conductor forward problem at small distances, as well as in the interpretation and simulation of tissue bath experiments in which the planar assumption is rigorously met [3,5-8]. As stated in previous work [9-11] describing the use of medium filters to solve volume conductor problems for idealized cylindrical geometries, 'The major impetus for the use of the medium filter method is in the reduction in computational complexity which results from such simplifying geometric assumptions.' Furthermore, the solution of two-dimensional volume conductor problems in which the source is a non-planar surface can easily be handled in rectangular coordinates by conformally mapping the surface into a plane [12].

The assumption of a layered medium is appropriate and useful for the proposed application of the algorithm: the study of the distortion of epicardial potentials in hearts damaged by patchy infarction, i.e. epicardial tissue with several discrete active layers separated by regions of fibrous growth. The modelling of these different regions as homogeneous layers is reasonable, since the smoothing and attenuation caused by minor inhomogeneities within a layer are far less significant than the smoothing caused by large conductivity discontinuities between layers (see $[10,11]$ and references therein). This explains why even the assumption of a homogeneous volume conductor, used extensively in previous work $[1,9-11,13-17]$ has given reasonable results.

This paper presents an exact solution to the forward and inverse two-dimensional volume conductor problems for a inhomogeneous layered conducting medium. By 'exact' we mean that all effects of Laplace's equation are included; arbitrary accuracy can be obtained in the absence of noise by using sufficiently finely sampled data. The volume conductor is assumed to consist of distinct layers of arbitrary conductivities and thicknesses. This is the first exact solution for this problem. The inverse problem can also be solved, although regularization using a special spatial low-pass filter is required.

\subsection{Previous results}

The one-dimensional volume conductor problem has been extensively described in the literature [1,15-17], using a variety of approaches. Ganapathy et al [9] proposed solutions to the one-dimensional forward and inverse problems by modelling the effect of the extracellular medium as an equivalent medium filter. They applied signal processing ideas to a formulation of the problem by Clark et al $[13,14]$. A medium filter was also used later for the radial geometry problem in $[10,11]$.

The medium filter approach formulates the expressions describing the desired potential as Fourier integrals. By subsequently approximating the continuous parameter 
Fourier transform as a discrete Fourier transform (DFT), these expressions can be rapidly computed using a fast Fourier transform (FFT) algorithm.

Multi-dimensional forward problems have been studied by several authors $[2,3,7]$. Spach et al [2] recently studied the two-dimensional case. They were able to relate the intercellular currents to the extracellular potentials by utilizing a discretization of the solution to an integral equation derived using Green theorem (discussed below). In [7], Geselowitz et al applied a finite-difference model to the problem discussed in [2]. They related the transmembrane current to extracellular voltage by modelling the medium as a network of resistors and computing the transfer impedance. By virtue of the finitedifference formulation, they were able to easily incorporate medium inhomogeneities into their solution.

\subsection{Inverse electrocardiography using epicardial potentials}

In [18] various electrocardiographic inverse problem solutions for inhomogeneous media are discussed. An extensive bibliography is also provided; the reader is referred to [18] for a summary of previous work on this problem. In all the cases discussed, a suitable forward problem solution method, such as an integral equation [2], or a finite element [19] formulation, is used to form a set of linear equations relating potentials on the surface of the heart (collected into a vector $V_{\text {heart }}$ ) to measured potentials (collected into the vector $\left.\boldsymbol{V}_{\text {measured }}\right)$ :

$$
V_{\text {measured }}=\mathbf{A} V_{\text {heart }} \text {. }
$$

The inverse problem is to solve the above system thus obtaining an expression relating $\boldsymbol{V}_{\text {heart }}$ to $\boldsymbol{V}_{\text {measured. }}$ It is pointed out in [18] that as a result of the ill-posedness of such inverse problems, $\mathbf{A}$ has a large condition number, resulting in an ill-conditioned and numerically unstable solution. Several regularization methods are also discussed.

In this study, we are concerned not with inverse electrocardiography, but with the recovery of fine potential features from measurements made close to the surface of the heart. Our work is broadly related to that discussed in [18] in that we also are faced with the inversion of a large ill-conditioned transfer matrix to obtain our inverse problem solution. Our approach differs, however, in that we solve the forward problem in a manner facilitating inverse problem solutions; specifically, we model the medium as an equivalent filter. This approach allows both numerically simplified computations of the forward and inverse problems, and also a simple, physically intuitive regularization procedure.

\section{Problem statement}

The volume conductor forward and inverse problems can be stated as follows. Given a distributed electrical source buried in a conducting medium, the forward problem is to determine the resulting electrical potential distribution in the surrounding medium. The inverse problem is to determine the characteristics of the source from electrical potential measurements made in the conducting medium.

We make the following assumptions:

1. The extracellular medium consists of homogeneous, passive, layers of infinite lateral extent, and arbitrary conductivities and thicknesses. 
2. The problem can be considered to be quasistatic, implying that the medium has negligible react ance and propagation delay. Therefore, all potentials are computed for an instant in time. For time-varying signals, the procedure can be repeated for each time sample desired. The quasistatic assumption ensures that each sample will be independent of the other samples [20].

3. The only source for the extracellular potential is the surface of the active tissue. The source potential is described by a function $s(x, y)$ and lies in a plane parallel to the layers of the extracellular medium.

Bioelectrical sources have also been modelled as current source densities [3], transmembrane potentials [9], as well as evoked extracellular potentials, as assumed above. These formulations can be related to each other: evoked extracellular potentials and transmembrane potentials have been related to current source densities by Clark and Plonsey $[13,20]$, among others. Linearity of the medium implies that a given source type corresponds to a unique source of each other type, to within an additive constant $[9,20]$.

This paper uses extracellular potentials in its presentation; current source densities or transmembrane potential source representations can also be used. However, use of the latter two source models requires an additional assumption: the active tissue must be assumed to act as a syncytium. This equivalent cell approach has been used $[3,9,13,16]$; the assumption is reasonable if individual cell contributions to the source functions need not be considered separately. Otherwise, the only difference is that the medium filters for transmembrane potentials and current densities are obtained from the medium filter for extracellular potentials by multiplication by an additional function (see appendixes $\mathrm{B}$ and $\mathrm{C}$ in [21]).

\section{Medium filter solution}

\subsection{Introduction}

Here the forward and inverse problems are solved by representing the effects of the medium as an equivalent filter, generalizing the approach of $[9,13,14]$ to the multidimensional case.

Let $x$ and $y$ be lateral coordinates and $z$ be the vertical coordinate perpendicular to the layer interfaces, with the planar source $s(x, y)$ located at $z=0$. Since the medium is homogeneous and has infinite extent in $x$ and $y$, the Green function relating the source $s(x, y)$ to the potential $\phi(x, y, z)$ at an arbitrary location has the form $(2 \pi / z) h\left(x-x_{0}, y-y_{0}, z\right)$. Hence

$$
\phi(x, y, z)=h(x, y, z) * * s(x, y)
$$

where ** denotes convolution.

Within each layer, the potential $\phi(x, y, z)$ generated by the source satisfies Laplace's equation, $\nabla^{2} \phi(x, y, z)=0$ with suitable boundary conditions (see subsection 3.3 below). In the frequency domain, the right-hand side of (2) becomes

$$
\phi(x, y, z)=\frac{1}{(2 \pi)^{2}} \iint S\left(k_{x}, k_{y}\right) H\left(k_{x}, k_{y}, z\right) e^{-\mathrm{i} k_{x} x} \mathrm{e}^{-\mathrm{i} k_{y} y} \mathrm{~d} k_{x} \mathrm{~d} k_{y}
$$


where $H\left(k_{x}, k_{y}, z\right)$ and $S\left(k_{x}, k_{y}\right)$ are the two-dimensional Fourier transforms of $h(x, y, z)$ and $s(x, y)$, respectively. Substituting (3) into Laplace's equation gives

$0=\iint S\left(k_{x}, k_{y}\right)\left[\frac{\partial^{2}}{\partial z^{2}} H\left(k_{x}, k_{y}, z\right)-\left(k_{x}^{2}+k_{y}^{2}\right) H\left(k_{x}, k_{y}, z\right)\right] \mathrm{e}^{-\mathrm{i} k_{x} x} \mathrm{e}^{-\mathrm{i} k_{y} y} \mathrm{~d} k_{x} \mathrm{~d} k_{y}$.

For a non-trivial (non-zero) surface potential solution to exist, the square-bracketed expression in the integrand must be zero, which implies

$$
\frac{\mathrm{d}^{2}}{\mathrm{~d} z^{2}} H\left(k_{x}, k_{y}, z\right)=\left(k_{x}^{2}+k_{y}^{2}\right) H\left(k_{x}, k_{y}, z\right)
$$

The general solution to (5) is

$$
H\left(k_{x}, k_{y}, z\right)=A_{i} \exp \left[-z\left(k_{x}^{2}+k_{y}^{2}\right)^{1 / 2}\right]+B_{i} \exp \left[z\left(k_{x}^{2}+k_{y}^{2}\right)^{1 / 2}\right]
$$

where $A_{i}$ and $B_{i}$ are distinct constants for each layer to be determined from the boundary conditions.

\subsection{Derivation of the medium filter for anisotropic media}

The medium filter for a homogeneous anisotropic medium can be derived by transforming this problem into an equivalent isotropic medium problem. Consider a layer with a conductivity matrix of the form

$$
\sigma=\left[\begin{array}{ccc}
\sigma_{x} & 0 & 0 \\
0 & \sigma_{y} & 0 \\
0 & 0 & \sigma_{z}
\end{array}\right] .
$$

The electric field $\boldsymbol{E}(x, y, z)$ is related to the potential $\phi(x, y, z)$ by $\boldsymbol{E}=-\nabla \phi(x, y, z)$, and Ohm's law is $J(x, y, z)=\sigma E(x, y, z)$, where $J(x, y, z)$ is current density. Since the extracellular region is assumed to be source free, we have

$$
\sigma_{x} \frac{\partial^{2} \phi}{\partial x^{2}}+\sigma_{y} \frac{\partial^{2} \phi}{\partial y^{2}}+\sigma_{z} \frac{\partial^{2} \phi}{\partial z^{2}}=\nabla \cdot J=\nabla \cdot(\sigma(-\nabla \phi))=0
$$

Now if we make the coordinate transformation

$$
x^{\prime}=\left(\sigma_{y} \sigma_{z}\right) x \quad y^{\prime}=\left(\sigma_{x} \sigma_{z}\right) y \quad z^{\prime}=\left(\sigma_{x} \sigma_{y}\right) z
$$

then $\phi^{\prime}\left(x^{\prime}, y^{\prime}, z^{\prime}\right)=\phi(x, y, z)$ satisfies Laplace's equation $\nabla^{2} \phi^{\prime}\left(x^{\prime}, y^{\prime}, z^{\prime}\right)=0$.

At this point we have reduced the anisotropic medium problem to the isotropic medium problem considered earlier. Hence the procedures used for isotropic media can also be used on anisotropic media, by simply scaling the coordinate axes. 


\subsection{Boundary conditions}

The boundary conditions for a multi-layered extracellular medium are as follows. At the peripheries of the medium we have

$$
\begin{aligned}
& H\left(k_{x}, k_{y}, 0\right)=1 \\
& H\left(k_{x}, k_{y}, \infty\right)=0
\end{aligned}
$$

i.e. the medium potential must agree with the surface potential at $z=0$, and tend to zero as $z \rightarrow \infty$.

At the $n$th interface $z=z_{n}$ between two conducting layers with conductivities $\sigma_{n}$ and $\sigma_{n-1}$, we have the following two additional boundary conditions (for $\delta \rightarrow 0$ ):

$$
\begin{aligned}
& H\left(k_{x}, k_{y}, z_{n}-\delta\right)=H\left(k_{x}, k_{y}, z_{n}+\delta\right) \\
& \sigma_{n-1} \frac{\partial H\left(k_{x}, k_{y}, z_{n}-\delta\right)}{\partial z}=\sigma_{n} \frac{\partial H\left(k_{x}, k_{y}, z_{n}+\delta\right)}{\partial z}
\end{aligned}
$$

i.e. the medium potential and normal current density must be continuous across an interface.

\subsection{Recursive computation of filter coefficients}

Substituting the general form of the medium filter (6) into the boundary conditions (12) and (13) results in the following recursion relating the filter coefficients $A_{n}$ and $B_{n}$ in a given layer to those in the layer below:

$$
\left[\begin{array}{l}
A_{n-1} \\
B_{n-1}
\end{array}\right]=\sqrt{\frac{\sigma_{n}}{\sigma_{n-1}}} \frac{1}{\sqrt{1-\Gamma_{n}^{2}}}\left[\begin{array}{cc}
1 & a_{n} \Gamma_{n} \\
\Gamma_{n} / a_{n} & 1
\end{array}\right]\left[\begin{array}{l}
A_{n} \\
B_{n}
\end{array}\right]
$$

Here $z=z_{n}$ is the interface between the $n$th and $(n-1)$ th layers, the interface reflection coefficient $\Gamma_{n}$ is defined as

$$
\Gamma_{n}=\frac{\sigma_{n-1}-\sigma_{n}}{\sigma_{n}+\sigma_{n-1}}
$$

and the layer attenuating factor $a_{n}$ is defined as

$$
a_{n}=\exp \left[2\left(k_{x}^{2}+k_{y}^{2}\right)^{1 / 2} z_{n}\right]
$$

\subsection{Initialization of recursion using linearity}

Define the outermost layer of the medium as the $N$ th layer, and the innermost layer of the medium, just above the planar source, as the zeroth layer. To initialize the recursion (14), we utilize the linearity of the volume conductor. The $N$ th layer may be a non-ideal conductor (with finite non-zero conductivity), a perfect conductor, or a perfect insulator. We consider each case separately. 
3.5.1. Non-ideal conductor. Assume the top ( $N$ th) layer has finite, non-zero conductivity $\sigma_{N}$, and has the form $z_{N}<z<\infty$. $B_{N}$ is known to be zero by (11). $A_{N}$ which is needed to initialize the recursion, is unknown; however, if (14) is initialized (incorrectly) using $A_{N}=1$ and $B_{N}=0$, the resulting computed $A_{0}$ and $B_{0}$ will be incorrect by a factor of $A_{N}$.

By equation (10) we know that $A_{0}+B_{0}=1$. However, the incorrectly computed $A_{0}+B_{0}$ will be $1 / A_{N}$, not 1 , so that the incorrectly computed $A_{0}+B_{0}$ specifies precisely the factor $A_{N}$ by which the computed $A_{n}$ and $B_{n}$ are in error.

Therefore the recursion (14) is initialized using

$$
A_{N}=1 \quad B_{N}=0 \text {. }
$$

Then proceed as follows:

1. Propagate (14) in decreasing $i$, computing $A_{i}$ and $B_{i}$ in each layer. These $A_{i}$ and $B_{i}$ will be incorrect by a factor $C$ (which is the correct $A_{N}$ ).

2. Compute $C=1 /\left(A_{0}+B_{0}\right)$.

3. Compute the correct $A_{i}$ and $B_{i}$ in each layer by multiplying the previously computed values by $C$.

3.5.2. Perfect conductor. Now assume the outermost layer is a perfect conductor $\left(\sigma_{N} \rightarrow \infty\right)$. In this case, it is necessary to initialize (14) in the $(N-1)$ th layer (the first layer below the top layer). Since the potential inside a perfect conductor is constant, equations (6) and (11) show that $A_{N, j}=B_{N, j}=0$. Inserting this into equation (6) and then inserting the result into equation (12) with $n=N-1$ yields

$$
B_{N-1}=-A_{N-1} a_{N}
$$

The recursion (14) is initialized in the $(N-1)$ th layer using $A_{N-1}=1$ and $B_{N-1}=$ $-a_{N}$. Then proceed as in the non-ideal conductor case above.

3.5.3. Perfect insulator. Now assume the outermost layer is a perfect insulator $\left(\sigma_{N}=\right.$ $0)$. In this case it is also necessary to initialize (14) in the $(N-1)$ th layer. Setting $n=N-1$ and $\sigma_{N}=0$ in (13) yields

$$
B_{N-1}=A_{N-1} a_{N}
$$

The recursion (14) is initialized in the (N-1)th layer using $A_{N-1}=1$ and $B_{N-1}=a_{N}$. Then proceed as in the non-ideal conductor case above. Note that the same procedure is used to initialize the recursion in each case, although the actual initial values (17), (18), and (19) differ.

\section{Medium filters for homogeneous media}

For homogeneous media, only two boundary conditions apply. As $z \rightarrow \infty$ the potential must tend to zero, so that $B=0$. At $z=0$ the medium potential must equal the surface potential, so that $H\left(k_{x}, k_{y}, 0\right)=1$ and $A=1$. 


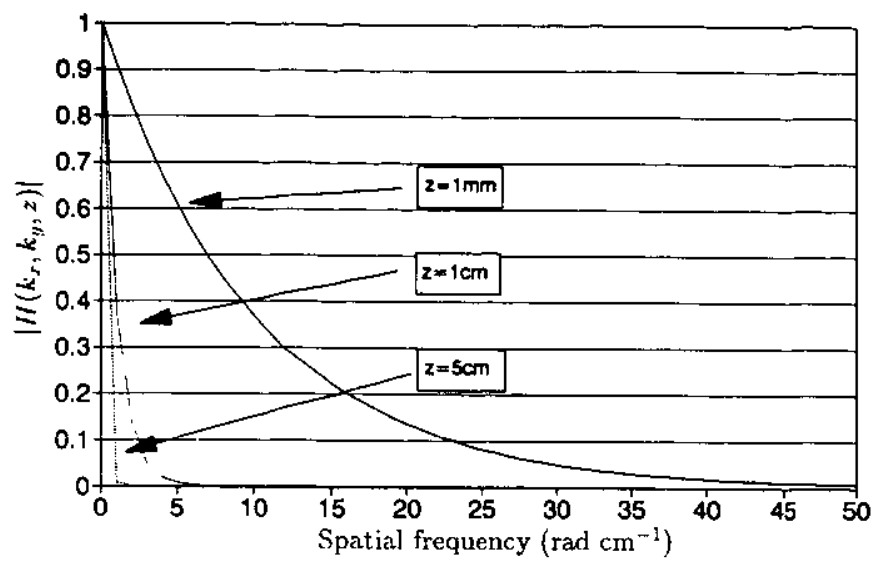

Figure 1. The response of the medium filter versus spatial frequency, at selected distances. Note that as the distance from the source increases, the attenuation of the medium filter becomes more severe. Spatial frequencies of up to about $25 \mathrm{rad} \mathrm{cm}^{-1}$ are required to accurately reproduce the test waveform given in figure 2.

Then the medium filter for a homogeneous medium has the form

$$
H\left(k_{x}, k_{y}, z\right)=\exp \left[-\left(k_{x}^{2}+k_{y}^{2}\right)^{1 / 2} z\right]
$$

Another way of deriving (20) is as follows. For a homogeneous medium, the extracellular potential $\phi(x, y, z)$ is related to the source potential by $(3)$ with $H\left(k_{z}, k_{y}, z\right)$ given in (20). Since the solution to Laplace's equation is unique, equation (3) can also be derived by directly taking the two-dimensional Fourier transform of the extracellular potential expression using Green theorem

$$
\phi(x, y, z)=\frac{z}{2 \pi} \iint \phi\left(x^{\prime}, y^{\prime}\right) G\left(x, x^{\prime}, y, y^{\prime}, z\right) \mathrm{d} x^{\prime} \mathrm{d} y^{\prime}
$$

where for a homogeneous unbounded medium the Green function is

$$
G\left(x, x^{\prime}, y, y^{\prime}, z\right)=\left[\left(x-x^{\prime}\right)^{2}+\left(y-y^{\prime}\right)^{2}+z^{2}\right]^{-3 / 2}=\frac{2 \pi}{z} h\left(x-x^{\prime}, y-y^{\prime}, z\right) .
$$

The convolution (21) can also be implemented in the Fourier domain as (3), where $(20)$ is the 2D Fourier transform of $h(x, y, z)$ in (22).

This shows that the filtering approach is mathematically equivalent to the solution using Green theorem, and thus can be interpreted as a fast method for solving the integral equation (21). This can also be shown for the general layered case, although the Green function is more complicated than equation (22).

\subsection{Physical interpretation of the medium filters}

By examining the functional form of the homogeneous medium filter (20) the effect of the medium on the potential field can be easily illustrated and understood. A plot of the magnitude response of the filter for various values of $z$ is provided in figure 1 . Note that the medium filter is a low-pass filter in $k_{x}$ and $k_{y}$. Also, as $z$ increases, higher frequencies are attenuated more rapidly. This corroborates experimental results that volume conductors both attenuate and smooth the potential field. However, the medium filter provides specific numerical information on the amount of attenuation of features at a specific spatial frequency and distance, information that is not immediately apparent from (21). 
The exponential form of the filter shows that the inverse problem is ill-conditioned at large distances and at high spatial frequencies, since the field due to a given feature attenuates exponentially with distance and with spatial frequency. Consequently, no inverse problem solution procedure can recover high-spatial-frequency information (i.e. fine detail), except at small distances.

By viewing the potential distribution as an evanescent wave field, one can readily see the effect of the conductivity discontinuities on the extracellular potential. The terms in the general solution (6) in each layer can be identified with evanescent waves $A \mathrm{e}^{-k z}$ attenuating with increasing depth $z$, and $B \mathrm{e}^{k z}$ growing with increasing depth $z$. The effect of the medium discontinuities is that of a frequency-dependent, partiallyreflecting boundary. This observation explains the functional form of the reflection coefficients in (15), governing the coupling of the potential field from one region to the next.

If the top layer is the exterior of the tissue (i.e. the air), it is effectively an infinite half-space. Even in situations where no convenient top layer can be found, the potential field attenuates so rapidly that for distances of $10 \mathrm{~cm}$ or greater the field can be assumed to be zero, with an error bounded by $0.1 \%$ for spatial frequencies of greater than $1 \mathrm{rad} \mathrm{cm}^{-1}$. By examining the form (15) of the reflection coefficients, one can also observe a limitation of all solutions to volume conductor problems: potential fields cannot be resolved behind large conductivity discontinuities.

For example, from equation (14) it is apparent that if the $n$th medium has high conductivity (e.g. blood or salt water), or very low conductivity, the coupling between the layers approaches zero due to the $1 / \sqrt{1-\Gamma_{n}^{2}}$ term. This situation results in a poorly-conditioned inverse problem, since the large conductivity discontinuity dramatically attenuates the extracellular potential.

\section{Implementation of the medium filter}

The Fourier transform $\Phi\left(k_{x}, k_{y}, z\right)$ of the potential $\phi(x, y, z)$ is related to the Fourier transform $S\left(k_{x}, k_{y}\right)$ of the source potential $s(x, y)$ by (3). The forward problem of computing $\phi(x, y, z)$ from $s(x, y)$ is solved by Fourier transforming $s(x, y)$, multiplying by $H\left(k_{x}, k_{y}, z\right)$, and then inverse Fourier transforming to get $\phi(x, y, z)$. Neglecting for the moment any regularization issues, the inverse problem of computing the source $s(x, y)$ from the potential $\phi(x, y, z)$ measured at a specific $z$ can be solved by Fourier transforming $\phi(x, y, z)$, multiplying by $1 / H\left(k_{x}, k_{y}, z\right)$, and then inverse Fourier transforming to get $s(x, y)$. Unfortunately, as will be discussed below, the inverse problem is generally ill-conditioned, which precludes the straightforward solution outlined here.

\subsection{Ill-posedness of the inverse problem}

Since the medium filter $H\left(k_{x}, k_{y}, z\right)$ has no finite zeros, the inverse medium filter $1 / H\left(k_{x}, k_{y}, z\right)$ is always defined. However, due to the exponential rolloff of the medium filter $H\left(k_{x}, k_{y}, z\right)$, as one moves away from the source the potential is both attenuated and smoothed. Consequently, the inverse filter $1 / H\left(k_{x}, k_{y}, z\right)$ is a high-pass filter, which amplifies fine detail to compensate for the effects of the intervening medium.

When computing inverse problem solutions in the presence of noise, the noise components at high spatial frequencies will be amplified exponentially relative to lowerfrequency components. Furthermore, due to the action of the forward filter, the signal power at high spatial frequencies will be weakest. The combination of these two effects 
makes it impractical to recover high-spatial-frequency features of the source potential, i.e. the inverse problem is ill-conditioned. Therefore, it is necessary to regularize the inverse problem, resulting in a smoothed reconstruction of the source potential.

Note that these properties are a result of the effects of the volume conductor itself, and would be present in any exact inverse problem solution-at high spatial frequencies and large distances, the inverse problem itself is ill-conditioned. This illconditioning appears in integral equation based procedures, since the integral equation kernel has a large condition number, resulting in a poorly-conditioned solution.

\subsection{Regularization and data filtering}

Now suppose that measurements are made in the presence of additive wide-band noise. Then the exponential characteristic of the inverse filter $1 / H\left(k_{x}, k_{y}, z\right)$ results in an estimate of the potential source corrupted with a noise whose power spectral density increases exponentially with spatial frequency over the bandwidth of the noise. Consequently, no polynomial-based low-pass filter, such as a Chebyschev filter, can roll off at the rate required to compensate for this exponential rise.

Ganapathy et al [9] also faced this problem in their one-dimensional medium filter inverse problem solution. They modeled the source potential as a random process, with power spectral density equal to the squared magnitude of the spectrum of the source potential, which was assumed to be known. A Wiener filter was then used as a low-pass filter.

Here a different Wiener filter is used. In this section this filter is derived using

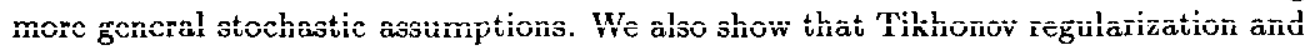
constrained least-squares regularization of the discretized integral equation of equation (21) also lead to the same filter.

\subsection{Formulation as a linear system of equations}

The problem is to reconstruct the source potential $s(x, y)$ from measurements of $\phi(x, y, z)$ for a known value of $z$. Recall that $\phi(x, y, z)$ is related to $s(x, y)$ by the integral equation (21). We now discretize this integral equation to obtain a linear system of equations relating discretized versions of $s(x, y)$ and $\phi(x, y, z)$.

The spatial variables $x$ and $y$ are discretized into $x=i \Delta$ and $y=j \Delta$. Define vectors $b$ and $x$ as the lexicographic orderings of the discretized $s(x, y)$ and $\phi(x, y, z)$ :

$$
\begin{aligned}
& \boldsymbol{b}=[\phi(0,0, z), \phi(0, \Delta, z), \cdots, \phi(0, j \Delta, z), \cdots, \phi(\Delta, 0, z), \cdots]^{\mathrm{T}} \\
& \boldsymbol{x}=[\boldsymbol{s}(\mathbf{0}, \mathbf{0}), \boldsymbol{s}(\mathbf{0}, \boldsymbol{\Delta}), \cdots, \boldsymbol{s}(\mathbf{0}, \mathbf{j} \boldsymbol{\Delta}), \cdots, \boldsymbol{s}(\boldsymbol{\Delta}, 0), \cdots]^{\mathrm{T}} .
\end{aligned}
$$

The kernel in (21) is discretized into a block-Toeplitz matrix $\mathbf{A}$

$$
\mathbf{A}=\left[\begin{array}{cccc}
H_{0} & H_{-1} & H_{-2} & \cdots \\
H_{1} & H_{0} & H_{-1} & \cdots \\
H_{2} & H_{1} & H_{0} & \cdots \\
\vdots & \vdots & \vdots & H_{0}
\end{array}\right]
$$

where each block $H_{j}$ is itself Toeplitz and defined as

$$
H_{j}=\left[\begin{array}{cccc}
h(j, 0) & h(j,-1) & h(j,-2) & \ldots \\
h(j, 1) & h(j, 0) & h(j,-1) & \ldots \\
h(j, 2) & h(j, 1) & h(j, 0) & \ldots \\
\vdots & \vdots & \vdots & h(j, 0)
\end{array}\right]
$$


where the function $h(i, j)$ is defined as

$$
h(i, j)=\frac{z \Delta^{2}}{2 \pi}\left((i \Delta)^{2}+(j \Delta)^{2}+z^{2}\right)^{-3 / 2} .
$$

for a homogeneous medium, and a more complicated expression for a layered medium. Now (21) can be written as the matrix equation $\boldsymbol{b}=\mathbf{A} \boldsymbol{x}$.

We now argue as follows:

1. The large Toeplitz-block-Toeplitz matrix $\mathbf{A}$ can be zero-padded into a circulantblock-circulant matrix, due to the symmetry of $\mathbf{A}$ and its blocks, which follows from $h(i, j)=h(i,-j)$ and $H_{j}=H_{-j}$ (see (27)). This is commonly performed in image restoration $([22],[23] \mathrm{p} 880)$.

2. The eigenvalues of the zero-padded $\mathbf{A}$ are the 2D DFT frequency spectrum of its first row $\tilde{\mathbf{A}}$, which is the discretized medium filter, lexicographically ordered. Its eigenvectors are the vectors implementing the DFT.

3. Since the zero-padded $\mathbf{A}$ is circulant-block-circulant, $\boldsymbol{b}=\mathbf{A} \boldsymbol{x}$ implements a circular 2D convolution. Alternatively, the eigendecomposition of $\mathbf{A}$ implements $\boldsymbol{b}=\mathbf{A} \boldsymbol{x}$ using the 2D DFT and the discretized medium filter.

4. Hence a discretized implementation of the medium filter using the DFT is equivalent to solving the (zero-padded) $\boldsymbol{b}=\mathbf{A} \boldsymbol{x}$.

Thus the inverse problem is to solve the ill-conditioned linear system of equations for $\boldsymbol{x}$

$$
\boldsymbol{b}=\mathbf{A} \boldsymbol{x} .
$$

The solution minimizing the error $\|\boldsymbol{b}-\mathbf{A} \boldsymbol{x}\|$ is the pseudoinverse $\hat{\boldsymbol{x}}_{\mathbf{P}}$ of $\mathbf{A}$ found by solving the square system

$$
\left(A^{\mathrm{T}} \mathbf{A}\right) \hat{x}_{\mathrm{P}}=\mathbf{A}^{\mathrm{T}} \boldsymbol{b}
$$

However, since $\mathbf{A}^{\mathrm{T}} \mathbf{A}$ is also ill-conditioned, a small perturbation in the data $\boldsymbol{b}$ will greatly perturb the pseudoinverse $\hat{x}_{P}$ Thus (29) must be regularized.

5.3.1. Tikhonov regularization. Tikhonov regularization [24] replaces minimization of the error $\|\boldsymbol{b}-\mathbf{A} \boldsymbol{x}\|$ with minimization of

$$
E=\|\boldsymbol{b}-\mathbf{A} \hat{x}\|^{2}+\gamma\|\hat{x}\|^{2}
$$

which puts a premium on not allowing the small singular values of $\mathbf{A}$ to make $\hat{\boldsymbol{x}}$ too large. Minimization of this functional replaces (29) with

$$
\left(\mathbf{A}^{\mathrm{T}} \mathbf{A}+\gamma \mathbf{l}\right) \hat{\boldsymbol{x}}=\mathbf{A}^{\mathrm{T}} \boldsymbol{b}
$$

where $\mathbf{I}$ is the identity matrix. This will clearly be a better-conditioned system than (29).

Zero-padding $\mathbf{A}$ into circulant-block-circulant form implies that $\mathbf{A}^{\mathbf{T}} \mathbf{A}$ is also circulant-block-circulant, so that their eigenvectors implement the 2D DFT, and their eigenvalues are their Fourier spectra. Therefore (31) can be written as

$$
\left(\left|\tilde{\mathbf{A}}_{i}\right|^{2}+\gamma\right) \tilde{\boldsymbol{x}}_{i}=\tilde{\mathbf{A}}_{i}^{*} \tilde{b}_{i}
$$


where $\tilde{\mathbf{A}}_{i}$ is the $i$ th element of the DFT of the first row $\tilde{\mathbf{A}}$ of $\mathbf{A}, \tilde{x}_{i}$ is the $i$ th element of the DFT of the elements of the vector $\hat{\boldsymbol{x}}$, and similarly for $\tilde{b}_{i}$. The solution of this equation is

$$
\tilde{x}_{i}=\frac{\tilde{A}_{i}^{*}}{\left|\tilde{A}_{i}\right|^{2}+\gamma} \tilde{b}_{i}
$$

In the limit as $\gamma \rightarrow 0$, the Tikhonov solution $\hat{\boldsymbol{x}}$ approaches the pseudoinverse $\boldsymbol{x}_{\mathbf{P}}$, which tends to oscillate wildly and grow large since the inversion of $\mathbf{A}$ is ill-conditioned. As $\gamma \rightarrow \infty, \hat{\boldsymbol{x}} \rightarrow 0$. The problem is to choose an intermediate value of $\gamma$ that will produce a reasonable solution. The form of the error criterion (30) suggests that $1 / \gamma$ be chosen to be the signal-to-noise ratio. This follows since $\|\boldsymbol{b}-\mathbf{A} \boldsymbol{x}\|^{2}$ is the noise energy and $\|x\|^{2}$ is the signal energy; the units of $\gamma$ follow from dimensional considerations. Let $N=\|\boldsymbol{b}-\mathbf{A} \boldsymbol{x}\|^{2}$ be the noise energy and $S=\|\boldsymbol{x}\|^{2}$ be the signal energy. Choosing $1 / \gamma=S / N$ allows (33) to be rewritten as

$$
\tilde{\boldsymbol{x}}_{i}=\frac{S \tilde{\mathbf{A}}_{i}^{*}}{S\left|\tilde{\mathbf{A}}_{i}^{2}\right|+N} \tilde{\boldsymbol{b}}_{\boldsymbol{i}}
$$

which has the form of a Wiener filter for estimating a white 2D random field (lexicographically ordered into $\tilde{\boldsymbol{x}}$ ) with strength $S$ from observations (lexicographically ordered into $\tilde{b}$ ) made in uncorrelated 2D white noise with strength $N$.

5.3.2. Constrained least-squares regularization. Since the measurements are made in the presence of noise, it is unreasonable to expect the estimate $\hat{\boldsymbol{x}}$ determined from the noisy data $\boldsymbol{b}$ to be 'closer' to the actual $\boldsymbol{x}$ than a direct measurement of $\boldsymbol{x}$ would be in the presence of the same noise. In constrained least-squares regularization [25], we choose the $\hat{\boldsymbol{x}}$ which minimizes $\|\boldsymbol{C} \hat{\boldsymbol{x}}-\boldsymbol{d}\|$, where $\mathrm{C}$ is a matrix that acts as a stabilizing functional.

The constrained least-squares regularization procedure thus reduces to the minimization of $\|\boldsymbol{C} \hat{x}-d\|$ subject to the constraint that $\|A \hat{x}-b\|^{2}=\epsilon$. The solution, easily obtained using Lagrange multipliers, is

$$
\left(\lambda \mathbf{A}^{\mathrm{T}} \mathbf{A}+\mathbf{C}^{\mathrm{T}} \mathbf{C}\right) \hat{\boldsymbol{x}}=\lambda \mathbf{A}^{\mathrm{T}} \boldsymbol{b}+\mathbf{C}^{\mathrm{T}} \boldsymbol{d}
$$

where the Lagrange multiplier $\lambda$ is chosen so that $\|\mathbf{A} \hat{x}-\boldsymbol{b}\|^{2}=\epsilon$. In particular, the minimum-nortn solution is found by setling $C=\mathbf{I}$ and $d=0$. In this case, (35) reduces to (31) with $\gamma=1 / \lambda$.

We apply constrained least-squares regularization to the system $\boldsymbol{b}=\mathbf{A} \boldsymbol{x}$, where the vector of observations $\boldsymbol{b}$ has noise added to it. We wish to determine the $\hat{\boldsymbol{x}}$ which minimizes $\|\boldsymbol{b}-\mathbf{A} \hat{\boldsymbol{x}}\|$ such that $\|\hat{\boldsymbol{x}}\|^{2}<\epsilon$; note that this constrains the energy of the reconstructed potential $s(x, y)$ to be less than $\epsilon$. Noting the symmetry of (35) in $A$ and $\mathbf{C}$, and $\boldsymbol{b}$ and $\boldsymbol{d}$, the constrained minimum-norm least-squares solution to $\boldsymbol{b}=\mathbf{A} \boldsymbol{x}$ is identical to the Tikhonov solution with $\gamma=1 / \lambda$.

5.3.3. Wiener filtering. The problem is now reformulated as the stochastic problem of computing the linear least-squares estimate of $s(x, y)$, which is now modelled as a zero-mean white random field with power spectral density $S$, from observations of the potential $\phi(x, y, z)$, to which a zero-mean white noise field with power spectral 
density $N$ has been added. The linear least-squares estimate $\hat{s}(x, y)$ is computed from the noisy observations using the Wiener filter [26]

$$
\frac{S H^{*}\left(k_{x}, k_{y}, z\right)}{S\left|H\left(k_{x}, k_{y}, z\right)\right|^{2}+N}
$$

(note that since $H\left(k_{x}, k_{y}, z\right)$ is real, $H\left(k_{x}, k_{y}, z\right)=H^{*}\left(k_{x}, k_{y}, z\right)$ ). Multiplying (36) by $(1 / S) /(1 / S)$ shows that this is the same as Tikhonov and constrained minimum-norm least-squares regularization.

Therefore, the Wiener regularizing filter, derived under the stochastic assumptions that the source potential is a $2 \mathrm{D}$ white random field, and that a $2 \mathrm{D}$ white noise field is added to the observations, is identical to the Tikhonov and constrained leastsquares regularizing filters, which made no stochastic assumptions. The fact that three completely different regularizing procedures give rise to the same regularizing filter strongly suggests the use of that filter.

Note that the assumption that the source potential is a $2 \mathrm{D}$ white random field is reasonable: since the spectrum of the source is actually unknown, the independent frequency components of the random field are all weighted equally a priori. This is tantamount to making no assumptions on the form of the source potential--its value at one location has no influence on its value at another location.

The use of Wiener filters to regularize volume conductor inverse problem solutions has been employed previously $[9,11]$ in radial geometry. The source was modeled as a random field with power spectral density approximated as the squared magnitude of the spectrum of the source potential. We rejected this approach since it was felt that a priori knowledge of the squared magnitude of the spectrum of the source potential (what we are attempting to find) was an unreasonable requirement.

The only information needed to compute our regularizing Wiener filter (36) is: (1) the depth $z$ of the source (which is also required by the medium filter); and (2) an estimate of the signal-to-noise ratio $S / N$. The latter is computed as follows. The average signal-plus-noise power $S+N$ is computed by summing the squares of the data points. The average noise power $N$ is computed by summing the squares of the data points at a different time, at which the tissue is unexcited. Assuming that measurements are made in relatively low-noise environments $(S / N>10)$, the signalto-noise ratio $S / N$ can be approximated by $S / N \approx(S+N) / N$, which is valid for $S \gg N$.

In practice we are most interested in reconstructing the lower spatial frequencies of the source potential, for two reasons: (1) these are most important in specifying its form; and (2) these are least affected by the medium. Hence it is important that the regularizing filter be unbiased at low frequencies. To achieve this, the regularizing Wiener filter (36) should be multiplied by $1+N / S$, the reciprocal of its response at $\left(k_{x}, k_{y}\right)=(0,0)$, to ensure an unbiased estimate at $\left(k_{x}, k_{y}\right)=(0,0)$. This is a common practice in image processing [23].

The overall filter $O\left(k_{x}, k_{y}, z\right)$ relating the observed potential to the reconstructed source potential is a cascade of the regularizing filter (36) and the unbiasing factor $(1+N / S)$. Note that this is not cascaded with the inverse medium filter, since the effect of the medium was included in (36)). The result is

$$
O\left(k_{x}, k_{y}, z\right)=\frac{(S+N) H\left(k_{x}, k_{y}, z\right)}{S H^{2}\left(k_{x}, k_{y}, z\right)+N}
$$


where the average powers of the noise $N$ and source $S$ are computed as described above. Note that in the absence of noise $(N=0)$ equation (37) reduces to $O\left(k_{x}, k_{y}, z\right)=$ $1 / H\left(k_{x}, k_{y}, z\right)$, which is the ideal inverse medium filter, regardless of the value of $S$.

One additional advantage of using a filtering approach is that by evaluating the regularization filter for various values of $z$, spatial frequency, and signal-to-noise ratios, one can predict a priori what features of the source potential are recoverable reliably. Furthermore, when intrepreting the results of an inverse problem solution, the evaluation of the regularization filter can be useful in judging the possibility of the existence of higher-spatial-frequency components which are unobservable in the inverse problem solution.

\section{Issues in numerical implementation}

\subsection{Discretization isues}

To allow implementation on a digital computer, the above continuous-parameter filter must be reformulated as a discrete filter. The discrete filter $H_{d}(n, m, z)$ is obtained from $H\left(k_{x}, k_{y}, z\right)$ by sampling the latter at regular intervals in $k_{x}$ and $k_{y}$, closely enough to avoid spatial aliasing. This method of FIR filter design is called frequency sampling [27] Specifically,

$$
H\left(k_{x}, k_{y}, z\right)=H\left(n \Delta k_{x}, m \Delta k_{y}, z\right)=H_{d}(n, m, z)
$$

where the sampling intervals $\Delta k_{x}, \Delta k_{y}$ must be no greater than

$$
\Delta k_{x}<\frac{\pi}{s_{x}} \mathrm{rad} \mathrm{cm}^{-1} \quad \Delta k_{y}<\frac{\pi}{s_{y}} \mathrm{rad} \mathrm{cm}^{-1} .
$$

Here $s_{x}$ and $s_{y}$ are the spatial extents in $\mathrm{cm}$ of the source potential in the $x$ and $y$ directions, respectively. Note that the Nyquist sampling rate accounts for the numerators of $\pi$ instead of $2 \pi$.

If the surface potential $\Phi\left(k_{x}, k_{y}, z\right)$ is measured over a grid with mesh cell size $\epsilon_{x}$ by $\epsilon_{y}$, then we also require that the discrete spatial Fourier transforms have orders

$$
N_{x}>2 s_{x} / \epsilon_{x} \quad N_{y}>2 s_{y} / \epsilon_{y} .
$$

The factors of two in (40) are present since the filtering operations are performed in the discrete frequency domain. Hence a circular convolution of the periodic extensions of $s(x, y)$ and $h(x, y, z)$ is computed, rather than the linear convolution desired in (2). To make the computed circular convolution equal to the desired linear convolution, the original sequences must be zero-padded to at least twice their spatial extent in both $x$ and $y$.

A power-of-two 2D FFT algorithm can be used by zero-padding the required transform orders $2 s_{x} / \epsilon_{x}$ and $2 s_{y} / \epsilon_{y}$ to the next higher power of two. Note that if the entire mesh has size greater than $s_{x}$ by $s_{y}$, then: (1) spatial aliasing will be avoided; (2) the resolution of the source potential will be the same as the mesh cell size; and (3) equation (2) will be accurately computed. 


\subsection{Measurement issues}

The actual potential to be reconstructed is a waveform moving in space over time. Measurements are assumed to be made by a brush electrode oriented perpendicular to the direction of propagation of the waveform. The temporal measurements at this specific location are then mapped into a similar waveform with spatial, rather than temporal, extent, by setting time equal to the $x$-coordinate divided by wave speed $(t=x / c)$. This is permissible due to the quasistatic assumption [20]. We assume here the direction of propagation is known, from measurements along two orthogonal lines [5]; without loss of generality we select the $x$-axis along this direction. If it is unknown, the wave speed can be determined by measurements at two different spatial locations, as described in [5].

For example, a ventricular action potential lasting $300 \mathrm{~ms}$ and propagating at $2 \mathrm{~m} \mathrm{~s}^{-1}$ (these are typical values [20]) would have a spatial extent of $(300 \mathrm{~ms}) \times$ $\left(2 \mathrm{~m} \mathrm{~s}^{-1}\right)=60 \mathrm{~cm}$. Note that this is greater than the spatial extent of cardiac tissue; this simply reflects the fact that the entire waveform does not appear simultaneously in space. To spatially sample this waveform every $0.5 \mathrm{~cm}$ would require a temporal sampling rate of the brush electrode signal of $\left(2 \mathrm{~m} \mathrm{~s}^{-1}\right) /(0.5 \mathrm{~cm})=400 \mathrm{~Hz}$.

The sensitivity of the inverse problem solution to noise suggests that measurements should be finely quantitized (typically eight bits or greater) to minimize the effects of quantitization noise.

\section{Numerical results}

To evaluate the new procedure, several simulated experiments were conducted. Experimental data were generated by solving the forward problem at various distances, and corrupting the solutions with additive white Gaussian noise. The use of additive white Gaussian noise is common in the literature [9-11]; it is used here to: (1) facilitate comparison with other papers on volume conductor problems; and (2) permit easy confirmation of the results by different researchers.

The noise-corrupted data was then used as input for the inverse problem procedure. A test waveform simulating the extracellular potential of a sheet of cardiac muscle tissue was utilized. The test action potential was designed to emphasize its rising edge, which is the most easily observed feature in the extracellular space.

\subsection{Test waveform}

The test action potential, a modification of one given in [16], is shown in figure 2 . It represents a wave moving in the $+x$ direction, representing a situation where the tissue of interest is only active for specific regions in $y$. It describes an action potential with the following characteristics:

1. Duration $300 \mathrm{~ms}$ and wave speed $2 \mathrm{~m} \mathrm{~s}^{-1}$ This corresponds to a spatial extent of $60 \mathrm{~cm}$ in the direction of propagation (see above).

2. Extent of $4 \mathrm{~cm}$ perpendicular to the direction of propagation. This was chosen to be on the order of the size of the ventricle; we neglect effects of tissue curvature due to the severe attenuation of the volume conductor (see section 1).

3. Maximum repolarization rate of $200 \mathrm{~V} \mathrm{~s}^{-1}$.

The test waveform was sampled using $N_{x}=N_{y}=128$, at intervals of $\epsilon_{x}=0.5 \mathrm{~cm}$ and $\epsilon_{y}=0.3125 \mathrm{~mm}$, corresponding to spatial extents of $s_{x}=64 \mathrm{~cm}$ and $s_{y}=4 \mathrm{~cm}$ (the test waveform is zero outside the range shown in figure 2). 


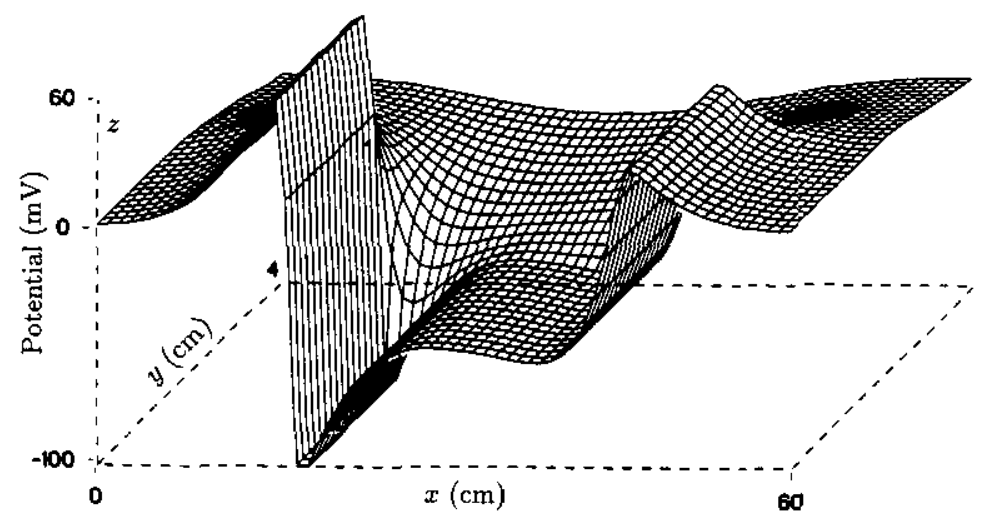

Figure 2. The 2D test function used as a source potential for all the simulated experiments. The region $0<y<2 \mathrm{~cm}$ represents the extracellular potential induced by activated cardiac muscle. The region $2 \mathrm{~cm}<y<4 \mathrm{~cm}$ represents inactive tissue. Since the test function represents a moving wave in the $x$ direction, such a waveform could be experimentally observed in cardiac tissue using a brush electrode and the techniques described in subsection 6.2.

\subsection{Discussion of simulation results}

Forward problem solutions were computed for distances of 1 and $5 \mathrm{~cm}$. In light of the proposed application of the algorithm, these distances are too large. They were chosen to illustrate the propertics of the volume conductor inver se piótientin, âs well as to test the limits of the inverse problem procedure. Two cases were considered: a homogeneous extracellular medium, and a layered extracellular media assumed to have three layers with characteristics as given in table 1 . Note that due to the form of equation (14) only relative conductivities are required.

Table 1.

\begin{tabular}{lll}
\hline Layer & Thickness (cm) & Relative Conductivity \\
\hline 1 & 2.5 & 1 \\
2 & 2.5 & 0.1 \\
3 & $\infty$ & 0 \\
\hline
\end{tabular}

The one-dimensional cross sections for the forward problem solutions in homogeneous media are displayed with the source potential in figure $3(a)$. This clearly displays the action of the volume conductor on the computed potential field. Note the additional attenuation and smoothing observed at $5 \mathrm{~cm}$ over that at $1 \mathrm{~cm}$.

The one-dimensional cross sections for the forward problem solutions in both homogeneous and layered media at $5 \mathrm{~cm}$ are illustrated in figure $3(b)$. The layer parameters are as in table 1 . Note the greater attenuation and smoothing of the source waveform due to the conductivity discontinuity, as compared to the homogeneous case.

Inverse problem solutions were computed for measurements made in homogeneous media at $0.1,0.5,1$ and $5 \mathrm{~cm}$, with signal-to-noise ratios $S / N=10,100$ and infinity (no noise). In the absence of noise, exact inverse problem solutions (no error) were obtained for all distances tested (and additionally up to about $100 \mathrm{~cm}$ ). In this case accuracy of the solutions was only limited by finite-register effects and overflow in 

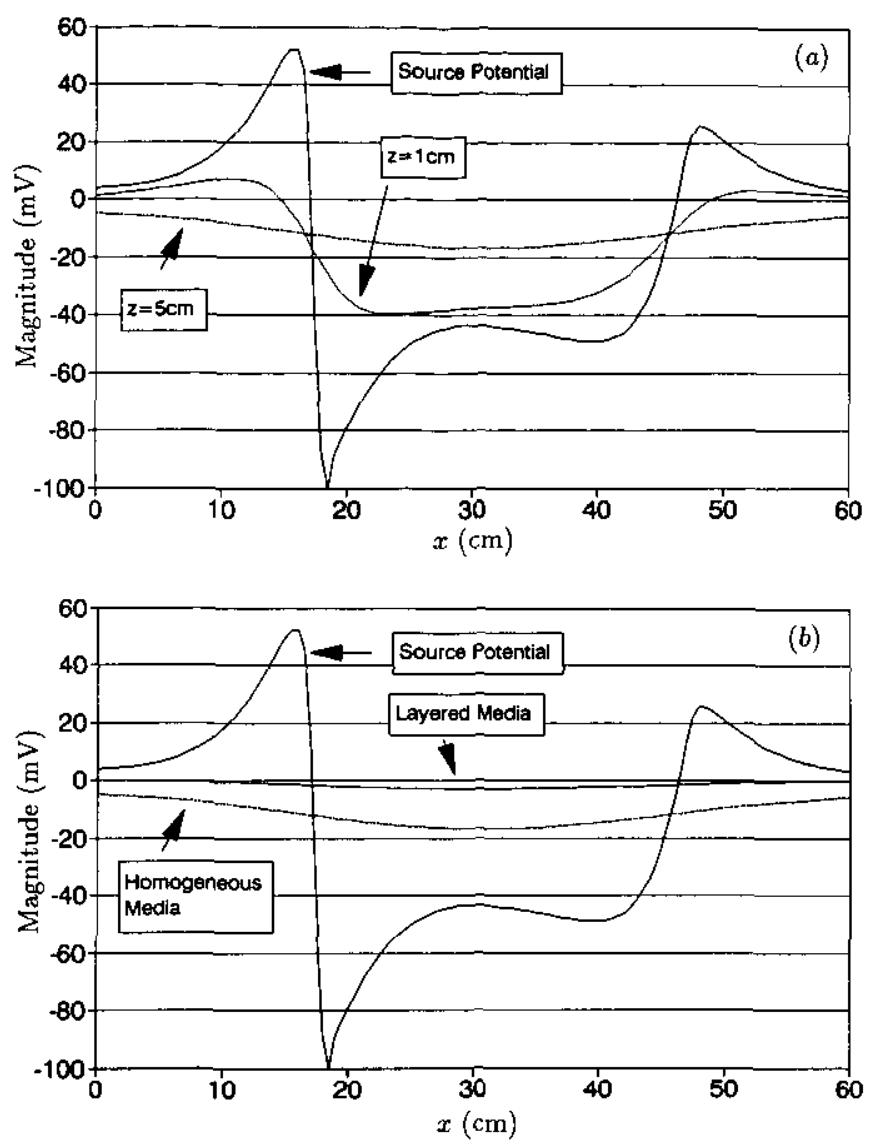

Figure 3. 1D cross sections (at $y=1 \mathrm{~cm}$ ) of the solution to the 2D forward problem. (a) at $z=0$, and $5 \mathrm{~cm}$, for both homogeneous and layered media. Note increased attemuation in the observed potential due to the conductivity discontinuity in the layered media. (b) At $z=0,1$, and $5 \mathrm{~cm}$ in a homogeneous medium. Note that the observed potential is both attenuated and smoothed as one moves away from the source.

the computations. Additionally, for distances of less than $1 \mathrm{~cm}$, the inverse problem solution was virtually exact for the above noise levels. This result is reasonable in light of the exponentially increasing attenuation of the volume conductor with distance. For these cases, no figures are given since the actual and reconstructed potentials coincide.

Figures $4(a)$ and $4(b)$ show the results for measurements made at $5 \mathrm{~cm}$ in homogeneous media with $S / N=10$ and 100 , respectively. These figures are one-dimensional cross sections, since the two-dimensional plots are difficult to interpret. For $S / N=10$ there is so much smoothing that only the general features of the potential are recoverable, although many of the features of the waveform (e.g. the point of activation and rising edge) are still visible. Therefore even in very high noise environments some important features of the waveform can still be resolved.

For $S / N=100$, the recovered potential is still significantly smoothed, but additional details, (e.g. higher spatial frequencies) are now present. This result can be expected in light of subsection 5.2, i.e. as the signal-to-noise ratio increases the inverse problem solution improves for higher spatial frequencies. Note that a measurement 

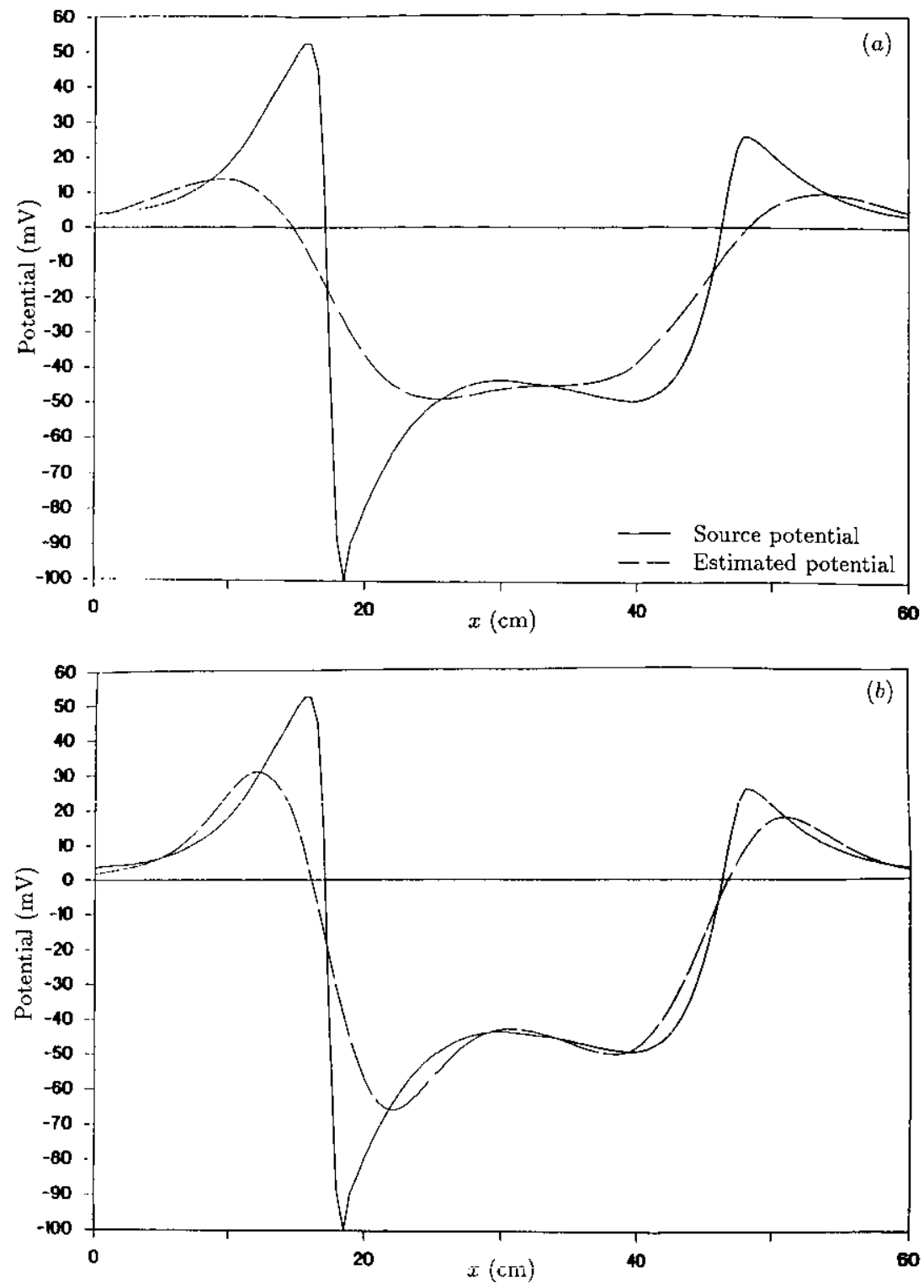

Figure 4. ID cross sections (at $y=1 \mathrm{~cm}$ ) of the solution to the $2 \mathrm{D}$ inverse problem at $z=5 \mathrm{~cm}$ in a homogeneous medium. (a) For $S / N=10$ there is so much smoothing that only the general features of the potential (e.g. the point of activation and rising edge) are still visible. (b) For $S / N=100$, the recovered potential is still significantly smoothed, but additional details (e.g. higher spatial frequencies) are now present.

distance of $5 \mathrm{~cm}$ is far greater than expected in our proposed application. Nonetheless, the inverse problem solution is still reasonable.

Figures $5(a)$ and $5(b)$ slow the results for measurements made at $1 \mathrm{~cm}$ with $S / N=$ 10 and 100 , respectively. At this distance, one can see that reasonable inverse problem solutions, exhibiting the form of the waveform, can be obtained even for $S / N=10$, while for $S / N=100$ the inverse problem solution is an excellent approximation to the 

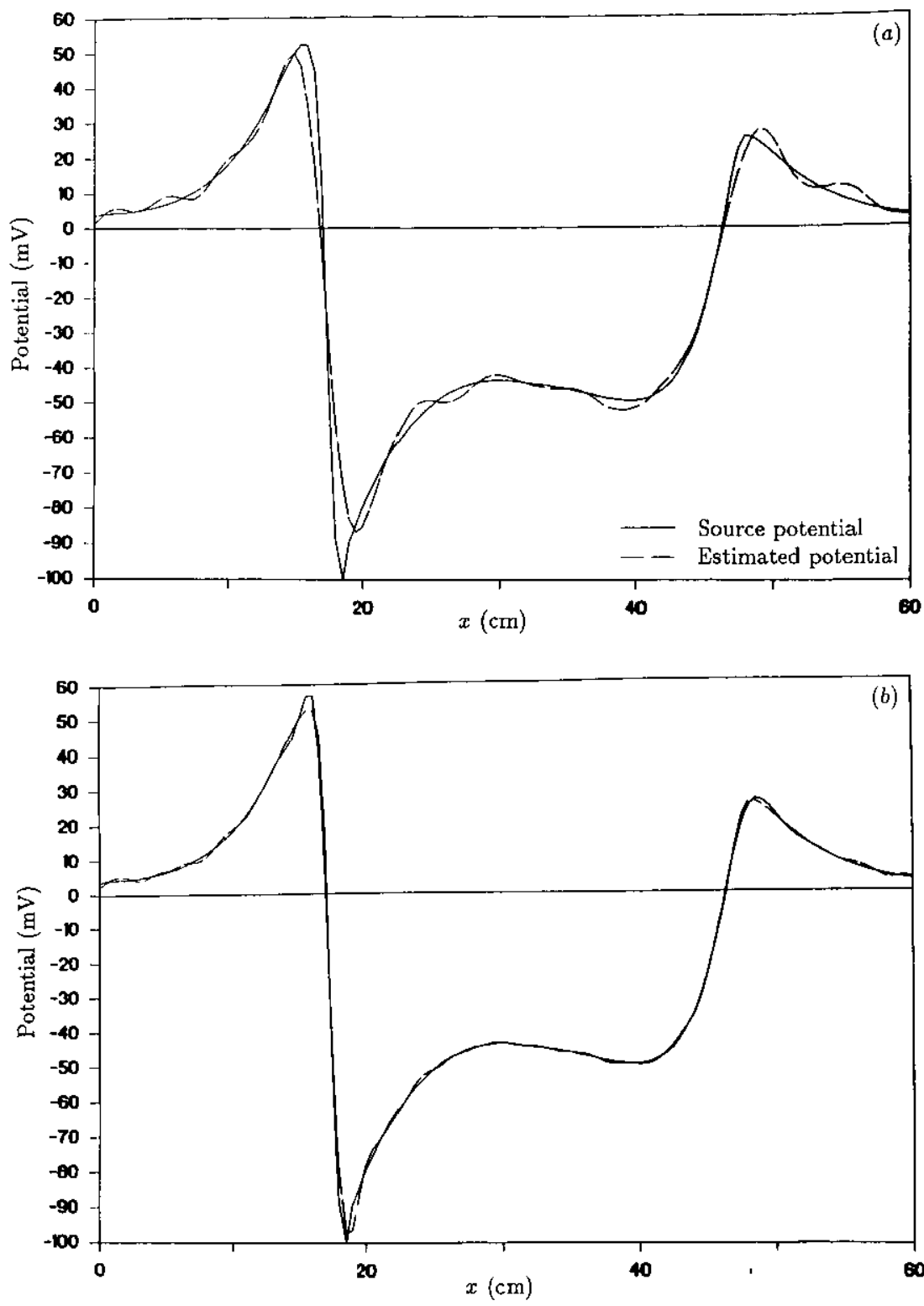

Figure 5. 1D cross sections (at $y=1 \mathrm{~cm}$ ) of the solution to the 2D inverse problem at $z=1 \mathrm{~cm}$ in a homogeneous medium. (a) $S / N=10$. Note that reasonable inverse problem solutions can be obtained even under this high noise condition. (b) $S / N=$ 100. At this noise level the inverse problem solution is an excellent approximation to the original source potential. In our proposed application this is a reasonable measurement distance.

original source potential.

For larger values of signal-to-noise ratio performance improves rapidly. Ganapathy and Clark, among others, have pointed out that 'experimentally, one is generally able to easily achieve signal-to-noise ratios that are larger than 100 by using high-quality differential amplifiers with adequate gain and good common-mode rejection (CMR) 
properties, coupled with a capacity for cumulative averaging to enhance the $S / N$ ratio' ([9] p 574). $60 \mathrm{~Hz}$ power line hum can also be reduced using adaptive filtering techniques [28].

\section{Conclusion}

A fast procedure for solving the two-dimensional volume conductor forward and inverse problems has been presented. It was shown that forward solutions can be computed quite readily, and inverse problem solutions can be obtained provided that the measurement distance is not too great and the signal-to-noise ratio is sufficiently high.

These solutions were obtained in a computationally efficient manner, by representing the extracellular medium as an equivalent filter, extending the work of $[9,13,14]$ to multi-dimensional sources. The filtering approach was also useful in interpreting the effects of the volume conductor on the potential field, and it also allowed the convenient use of regularization filters. The same regularization filter was derived using both stochastic and deterministic reasoning. In the stochastic approach we assumed that both the noise and the waveform to be reconstructed were white fields; thus no a priori knowledge about the waveform was required. In the deterministic approach we found that selecting the minimum-energy solution in the constrained least-squares regularization procedure and by using $S / N$ as the Lagrange multiplier in a Tikhonov regularization procedure resulted in the same regularization filter.

\section{Acknowledgments}

The first author would like to thank Professor C B Sharpe for his helpful consultation. The work of the first author was supported by the Office of the Vice-President for Research of the University of Michigan through an NIH grant. The work of the second author was supported in part by the National Science Foundation under grant no MIP-8858082.

\section{References}

[1] Spach M S, Barr R C, Johnson E A and Kootsey J M 1973 Cardiac extracellular potentials, analysis of complex wave forms about the Purkinje networks in dogs Circulation Res. 33 46.5-73

[2] Spach M S and Dolber P C 1986 Relating extracellular potentials and their derivatives to anisotropic propagation at a microscopic level in human cardiac muscle Circulation Res. 58 356-71

[3] Spach M S, Miller W T III, Miller-Jones E, Warren R B and Barr R C 1979 Extracellular potentials related to intracellular action potentials during impulse conduction in anisotropic canine cardiac muscle Circulation Res. 45 356-71

[4] Barr R C, Pilkington T C, Boineau J P and Spach M S 1966 Determining surface potentials from current dipoles, with application to electrocardiography IEEE Trans. Biamed. Eng. BME-13 88-92

[5] Kadish A H, Spear J F, Levine J H, Hanich R F, Prood C and Moore E N 1986 Vector mapping of myocardial activation Circulation 74 603-15

[6] Kadish A H, Moore E N, Levine J H, Blake C W and Spear J F 1988 Interactions of fiber orientation and direction of impulse propagation with anatomic barriers in anisotropic canine myocardium Circulation $\mathbf{7 8}$ 
[7] Geselowitz D B, Barr R C, Spach M S and Miller W T III 1982 The impact of adjacent isotropic fluids on electrograms from anisotropic cardiac muscle Circulation Res. 51 602-13

[8] Plonsey R and Barr R C 1987 Interstitial potentials and their change with depth into cardiac tissue Biophys. J. 51 547-55

[9] Ganapathy N, Clark J W, Wilson O B and Giles W 1985 Forward and inverse potential field solutions for cardiac strands of cylindrical geometry IEEE Trans. Biomed. Eng. BME-32 566-77

[10] Wilson O B, Clark J W, Ganapathy $N$ and Harman T 1985 Potential field from an active nerve in an inhomogeneous, anisotropic volume conductor-the forward problem IEEE Trans. Biomed. Eng. BME-32 1032-41

[11] Wilson O B, Clark J W, Ganapathy N and Harman T 1985 Potential field from an active nerve in an inhomogeneous, anisotropic volume conductor-the inverse problem IEEE Trans. Biomed. Eng. BME-32 1042-51

[12] Churchill R V and Brown J W 1984 Complex Variables and Applications (New York: McGrawHill)

[13] Clark J W and Plonsey R 1966 A mathematical evaluation of the core conductor model Biophys. J. 6 95-112

[14] Clark $J$ and Plonsey $R 1968$ The extracellular potential field of the single active nerve in a volume conductor Biophys. J. 8 842-64

[15] Geselowitz D B 1966 Comments on the core conductor model Biophys. J. 6 691-2

[16] Harmon T L, Liebfried T F, Clark J W and Hibbs C W 1975 A comparison of two methods for determining the extracellular potential field of an isolated Purkinje strand in a volume conductor IEEE Trans. Biomed. Eng. BME-22 174-83

[17] Greco E C and Clark J W 1977 The field from an isolated nerve in a volume conductor IEEE Trans. Biomed. Eng. BME-24 18-23

[18] Rudy $Y$ and Messinger-Rapport B J 1988 The inverse problem in electrocardiography: solutions in terms of epicardial potentials CRC Critical Rev. Biomed. Eng. 16 215-68

[19] Pilkington T C, Morrow M N and Stanley P C 1985 A comparison of finite elements and integral equation formulations for the calculation of electrocardiographic potentials $I E E E$ Trans. Biomed. Eng. BME-32 174-83

[20] Plonsey R 1969 Bioelectric Phenomena (New York: McGraw-Hil)

[21] Xydis T G, Yagle A and Kadish A 1990 Estimation of locations of bioelectric sources using an equivalent filter Inverse Problems 6 696-708

[22] Gray R M 1970 Toeplitz and circulant matrices: a review Technical Report Stanford University

[23] Beimond J, Lagendijk R L and Mersereau R M 1990 Iterative methods for image deblurring Proc. IEEE 78 856-83

[24] Tikhonov A N and Arsenin V Y 1977 Solutions of Mll-Posed Problems (New York: Wiley)

[25] Hunt B R 1973 The application of constrained least-squares estimation to image restoration by digital computer IEEE Trans. Comput. C-22 805-12

[26] Wiener N 1949 Extrapolation, Interpolation, and Smoothing of Stationary Time Series (New York: Wiley)

[27] Oppenheim A V and Schafer R W 1975 Digital Signal Processing (Englewood Cliffs, NJ: Prentice-Hall)

[28] Ferrara E R Jr and Widrow B 1981 The time-sequenced adaptive filter IEEE Trans. Acoustics, Speech, Signal Process. ASSP-29 679-83 